\title{
Communication
}

\section{Preventive Effects of Citrus unshiu Peel Extracts on Bone and Lipid Metabolism in OVX Rats}

\author{
Dong Wook Lim ${ }^{1}$, Youngseok Lee ${ }^{2}$ and Yun Tai Kim ${ }^{1, *}$ \\ 1 Functionality Evaluation Research Group, Division of Metabolism and Functionality Research, \\ Korea Food Research Institute, Seongnam 463-746, Korea \\ 2 Department of Bio \& Fermentation Convergence Technology, Kookmin University, \\ Seoul 136-702, Korea \\ * Author to whom correspondence should be addressed; E-Mail: ytkim@kfri.re.kr; \\ Tel.: +82-31-780-9295; Fax: +82-31-709-9876.
}

Received: 18 November 2013; in revised form: 6 January 2014 / Accepted: 7 January 2014 / Published: 9 January 2014

\begin{abstract}
Dried Citrus unshiu peel has been widely used for various medicinal purposes in Oriental Medicine. This study evaluated the metabolic effects of dried C. unshiu peel in ovariectomized (OVX) rats. The OVX rats were divided into five groups treated with distilled water, 17ß-estradiol (E2 $10 \mu \mathrm{g} / \mathrm{kg}$, once daily, i.p.) and dried C. unshiu peel extracts (DCPE 30, 100 and $300 \mathrm{mg} / \mathrm{kg}$, once daily, p.o.) for eight weeks. The treatments with high-dose DCPE significantly decreased the bone mineral density (BMD) loss in the femur, which was reflected by the decrease in alkaline phosphatase (ALP), telopeptides of collagen type I (CTx) and osteocalcin (OC) serum levels. It also inhibited the increase in lipoprotein levels compared to the OVX-control group without elevating the serum levels of estradiol, aspartate aminotransferase (AST) and alanine transaminase (ALT). Furthermore, DCPE exhibits a hepatoprotective effect in OVX-induced hepatic steatosis, indicated by reduced hepatic lipid contents. Taken together, our findings suggest that DCPE has the potential to improve both lipid and bone metabolism without influencing hormones such as estrogen in OVX rats.
\end{abstract}

Keywords: Citrus unshiu peel; anti osteoporosis; hepatoprotective effect 


\section{Introduction}

Menopause is often associated with serious public problems in middle-aged women; thus, appropriate care and management of health after menopause is important for maintaining a woman's quality of life [1]. A reduction in estrogen levels is commonly believed to cause psychological and mood changes, as well as physiological changes that result in symptoms such as osteoporosis, breast cancer, hot flashes, obesity, hyperlipidemia, and cardiovascular disease [2]. These menopausal symptoms can be at least comparatively reversed by the local or systemic administration of exogenous estrogens; thus, hormone replacement therapy (HRT) has been used to ameliorate menopausal symptoms [3]. However, long-term HRT increases the risk of several serious diseases, such as breast and endometrial cancer, thromboembolic events and vaginal bleeding [4]. Therefore, alternative approaches, including dietary interventions, have been interested in the treatment of menopausal symptoms.

Phytoestrogens are naturally occurring hormone-like compounds found in plants that can be subdivided into coumestans, isoflavonoids and lignans. Coumestrol, genistein, daidzein, and their plant precursors, are mainly found in soybeans and clover [5]. Isoflavones, especially derived from plants have various biological activities. They can improve metabolic symptoms [6] and bone-protective effects [7] by menopause. Although some studies have suggested that isoflavones have potential health benefits in postmenopausal women [8], a number of randomized trials have also failed to show a sustained enhancement of bone by isoflavone supplements. Since the evidence to support any beneficial effect of isoflavone intake on menopausal symptoms is not clear, making it is necessary to investigate the effects of natural agents other than isoflavones [9].

Citrus unshiu Marcov, which belongs to the family of Rutaceae, is a seedless and easy-peeling Korean citrus fruit that accounts for $30 \%$ of the total fruits produced in Korea. Its dried peels have been widely used as a folk medicine in Korea, China and Japan to improve bronchial and asthmatic conditions or blood circulation [10,11]. Dried C. unshiu peel has also been reported to process anti-inflammatory [12], anti-obesity [10], anti-allergic [13], hypolipidemic [14] and anti-cancer [15] effects. C. unshiu peel contains many phytochemicals such as hesperidin, naringin and nobiletin [16]. In particular hesperidin, the most abundant flavonoid in Citrus peel [17], has been shown to prevent bone loss in male orchidectomized rats following citrus juice consumption [18]. These reports led us to hypothesize that $C$. unshiu peel extracts could serve as a potent therapeutic agent for the menopausal symptoms, which often include dyslipidemia and osteoporosis. However, their efficacy needs to be scientifically evaluated in vivo experiments.

Here, we used OVX rats, which exhibit most of the characteristics of human menopausal symptoms [19]. They were treated with standardized dried C. unshiu peel extracts (DCPE) during an eight-week experimental period and then assessed the metabolic parameters related to menopausal symptoms.

\section{Results and Discussion}

\subsection{Analysis of Hesperidin from Dried Citrus unshiu Peel Extracts}

DCPE was monitored at $284 \mathrm{~nm}$ for hesperidin (Figure 1). The content of hesperidin was calculated for standardization. C. unshiu peel extracts was standardized to contain $21.7 \mathrm{mg} / \mathrm{g}$ hesperidin. 
Figure 1. 2-D HPLC chromatogram for standardization of DCPE.

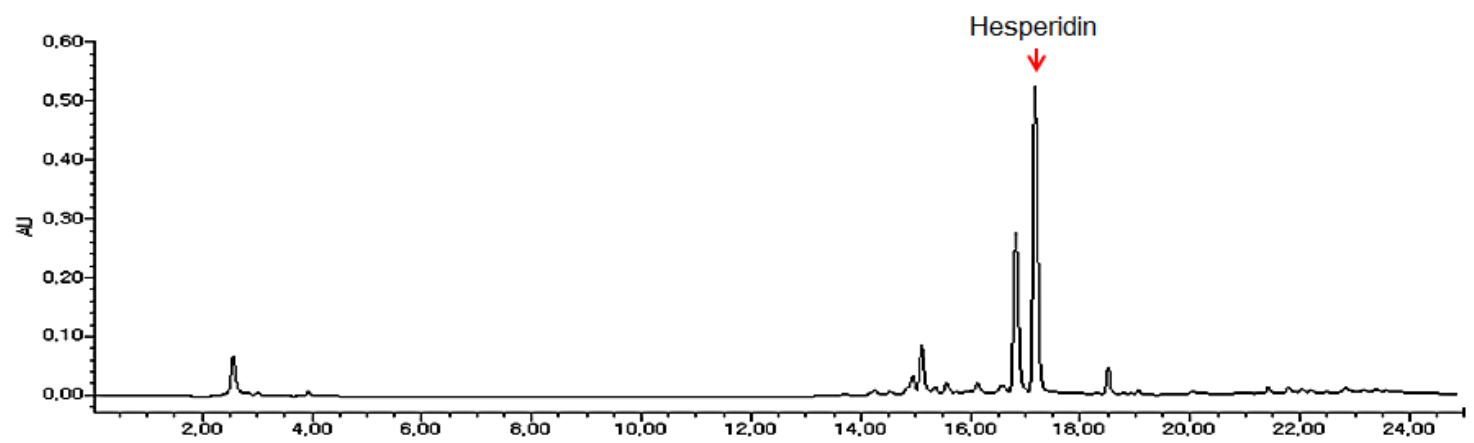

\subsection{Effect of DCPE on Body Weight and Serum Lipid Concentrations in OVX Rats}

The body weights in the OVX-control group statically increased compared to the sham group. As expected, a significant difference in body weight was observed between the E2 $10 \mu \mathrm{g} / \mathrm{kg}$ treated group and the OVX-control group by two weeks after initiating administration (Figure 2A). However, there was no significant difference in the body weight gain of the DCPE treated groups during the experimental period (Figure 2B). After eight weeks of treatments, the DCPE $300 \mathrm{mg} / \mathrm{kg}$ treated group showed significantly lower serum TC, TG and LDL-c levels, while causing the reverse on serum HDL-c. Similarly, DCPE $300 \mathrm{mg} / \mathrm{kg}$ caused significant reductions in the coronary artery risk and atherogenic indices (Table 1).

Figure 2. Effects of DCPE on weekly body weight (A) and body weight gain after eight weeks (B) in OVX rats. All data are the mean $\pm \mathrm{SD}(n=12$ per group). The asterisks indicated significant differences from the OVX-control group $(* p<0.05)$ based on one-way ANOVA and the Turkey post hoc test.
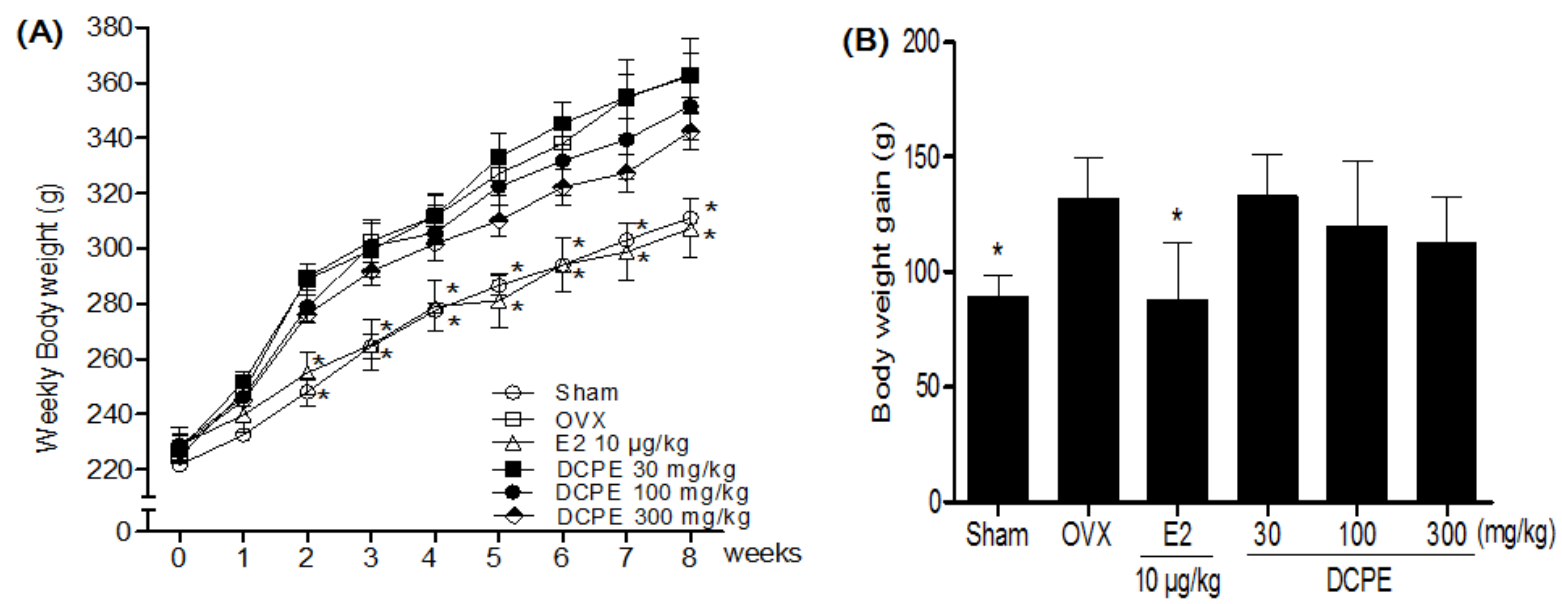

\subsection{Effect of DCPE on OVX-Induced Fatty Liver}

We found that DCPE exhibits a hepatoprotective effect in vivo, indicated by reduced hepatic lipid contents, serum AST and ALT levels (Table 2). It was found that OVX-control rats developed a high degree of steatosis, with severe cytoplasmic vacuoles and hepatocyte swelling (Figure 3B), whereas no 
histological abnormalities were observed in normal rats (Figure 3A). The treatments of DCPE 300 $\mathrm{mg} / \mathrm{kg}$ resulted in the prevention of hepatic fatty deposition in hepatocytes (Figure 3F).

Table 1. Effects of DCPE on serum lipoproteins in OVX rats.

\begin{tabular}{|c|c|c|c|c|c|c|}
\hline Groups & $\begin{array}{c}\mathrm{TC} \\
(\mathrm{mg} / \mathrm{dL})\end{array}$ & $\begin{array}{c}\text { TG } \\
(\mathrm{mg} / \mathrm{dL})\end{array}$ & $\begin{array}{c}\text { HDL-c } \\
(\mathrm{mg} / \mathrm{dL})\end{array}$ & $\begin{array}{c}\text { LDL-c } \\
(\mathrm{mg} / \mathrm{dL})\end{array}$ & CRI & AI \\
\hline I & $75.8 \pm 8.5$ & $78.5 \pm 5.2$ & $50.5 \pm 4.1$ & $15.7 \pm 6.1$ & $1.7 \pm 0.2$ & $0.3 \pm 0.2$ \\
\hline II & $97.4 \pm 5.1^{\mathrm{c}}$ & $98.3 \pm 6.4^{\mathrm{c}}$ & $41.5 \pm 2.3$ & $33.6 \pm 4.2^{\mathrm{c}}$ & $2.3 \pm 0.3^{c}$ & $0.8 \pm 0.2^{\mathrm{c}}$ \\
\hline III & $90.0 \pm 4.8$ & $85.7 \pm 8.7^{\mathrm{a}}$ & $44.8 \pm 4.3$ & $18.3 \pm 5.4^{\mathrm{a}}$ & $2.0 \pm 0.2^{\mathrm{a}}$ & $0.4 \pm 0.1^{\mathrm{a}}$ \\
\hline IV & $98.2 \pm 5.8$ & $90.2 \pm 5.4$ & $42.5 \pm 3.8$ & $27.3 \pm 4.2$ & $2.3 \pm 0.3$ & $0.6 \pm 0.1$ \\
\hline V & $92.0 \pm 5.4$ & $88.7 \pm 8.7$ & $42.5 \pm 4.8$ & $26.3 \pm 5.4$ & $2.2 \pm 0.2$ & $0.6 \pm 0.2$ \\
\hline VI & $85.0 \pm 6.5^{\mathrm{a}}$ & $80.9 \pm 5.6^{a}$ & $48.9 \pm 4.6$ & $17.9 \pm 4.8^{\mathrm{a}}$ & $1.7 \pm 0.2^{\mathrm{a}}$ & $0.4 \pm 0.3^{b}$ \\
\hline
\end{tabular}

Group I = Sham; Group II = OVX-control; Group III = E2 $10 \mu \mathrm{g} / \mathrm{kg}$; Group IV = DCPE $30 \mathrm{mg} / \mathrm{kg}$; Group $\mathrm{V}=$ DCPE $100 \mathrm{mg} / \mathrm{kg}$; Group VI = DCPE $300 \mathrm{mg} / \mathrm{kg}$. Data are mean $\pm \mathrm{SD}(n=12$ per group $) .{ }^{\mathrm{a}}$ A significant decrease at $p<0.05$, when compared with Group II values; ${ }^{\mathrm{b}} \mathrm{A}$ significant decrease at $p<0.01$, when compared with Group II values; ${ }^{\mathrm{c}}$ A significant increase at $p<0.05$, when compared with Group I values. CRI: coronary artery risk index, AI: atherogenic index.

Table 2. Effects of DCPE on OVX-induced hepatic steatosis.

\begin{tabular}{ccccc}
\hline \multirow{2}{*}{ Groups } & \multicolumn{2}{c}{ Serum $(\mathbf{m g} / \mathbf{d L})$} & \multicolumn{2}{c}{ Liver lipid (mg/g wet wt) } \\
\cline { 2 - 5 } & AST & ALT & TC & TG \\
\hline I & $115.1 \pm 11.6$ & $62.1 \pm 6.9$ & $6.1 \pm 0.7$ & $38.2 \pm 5.9$ \\
II & $144.4 \pm 25.7^{\mathrm{c}}$ & $96.5 \pm 6.6^{\mathrm{c}}$ & $10.1 \pm 0.7^{\mathrm{c}}$ & $69.8 \pm 6.4^{\mathrm{c}}$ \\
III & $121.2 \pm 17.4^{\mathrm{a}}$ & $72.5 \pm 5.6^{\mathrm{a}}$ & $7.0 \pm 0.5^{\mathrm{a}}$ & $54.5 \pm 6.2^{\mathrm{a}}$ \\
IV & $132.3 \pm 16.4^{\mathrm{a}}$ & $84.5 \pm 6.6$ & $8.2 \pm 0.6^{\mathrm{a}}$ & $70.5 \pm 4.2^{\mathrm{a}}$ \\
V & $123.1 \pm 16.4^{\mathrm{a}}$ & $75.3 \pm 4.9^{\mathrm{a}}$ & $7.2 \pm 0.6^{\mathrm{a}}$ & $52.8 \pm 6.8^{\mathrm{a}}$ \\
VI & $118.6 \pm 10.5^{\mathrm{a}}$ & $67.5 \pm 6.8^{\mathrm{a}}$ & $6.5 \pm 0.5^{\mathrm{a}}$ & $44.1 \pm 5.8^{\mathrm{a}}$ \\
\hline
\end{tabular}

The mean $\pm \mathrm{SD}$ of all data ( $n=12$ per group) are shown. ${ }^{\mathrm{a}} \mathrm{A}$ significant decrease at $p<0.05$, when compared with Group II values; ${ }^{\mathrm{c}} \mathrm{A}$ significant increase at $p<0.05$, when compared with Group I values.

\subsection{Effect of DCPE on BMD and Organ Weights in OVX Rats}

Three weeks after the OVX operation, OVX groups showed a significant decrease in the femur BMD compared to the sham group (Figure 4B). After eight weeks of treatments, the final femur BMD of the DCPE $300 \mathrm{mg} / \mathrm{kg}$ treated group was significantly higher than that of the OVX-control group (Figure 4A). OVX caused atrophy of uterine tissue, indicating the success of the surgical procedure and in the E2 $10 \mu \mathrm{g} / \mathrm{kg}$ treated group, the uterus index $(\mathrm{mg} / \mathrm{g})$ increased significantly compared to the OVX-control group. However, the DCPE treated groups did not show an effect on the uterus index following OVX operation (Figure 4C). In addition, the index of heart, liver, spleen and kidney was not significantly different in each group; either (Figure 4D). 
Figure 3. Histological images of liver tissues from OVX-induced hepatic steatosis displaying the hepatoprotective effects of DCPE. There were no histological abnormalities observed in sham rats (A). OVX-induced hepatic steatosis showing hepatocytes with severe cytoplasmic vacuoles and swelling (B). E2 $10 \mu \mathrm{g} / \mathrm{kg}$ resulted in the prevention of hepatic fatty deposition in hepatocytes (C). The treatments with DCPE 30, 100 and $300 \mathrm{mg} / \mathrm{kg}$ resulted in the prevention of hepatic fatty deposition in hepatocytes (D, $\mathbf{E}$ and $\mathbf{F})$. The tissues were surgically excised and subjected to histological study by staining with hematoxylin and eosin $(\mathrm{H} \& \mathrm{E})$. The arrows indicate fatty hepatocytes.

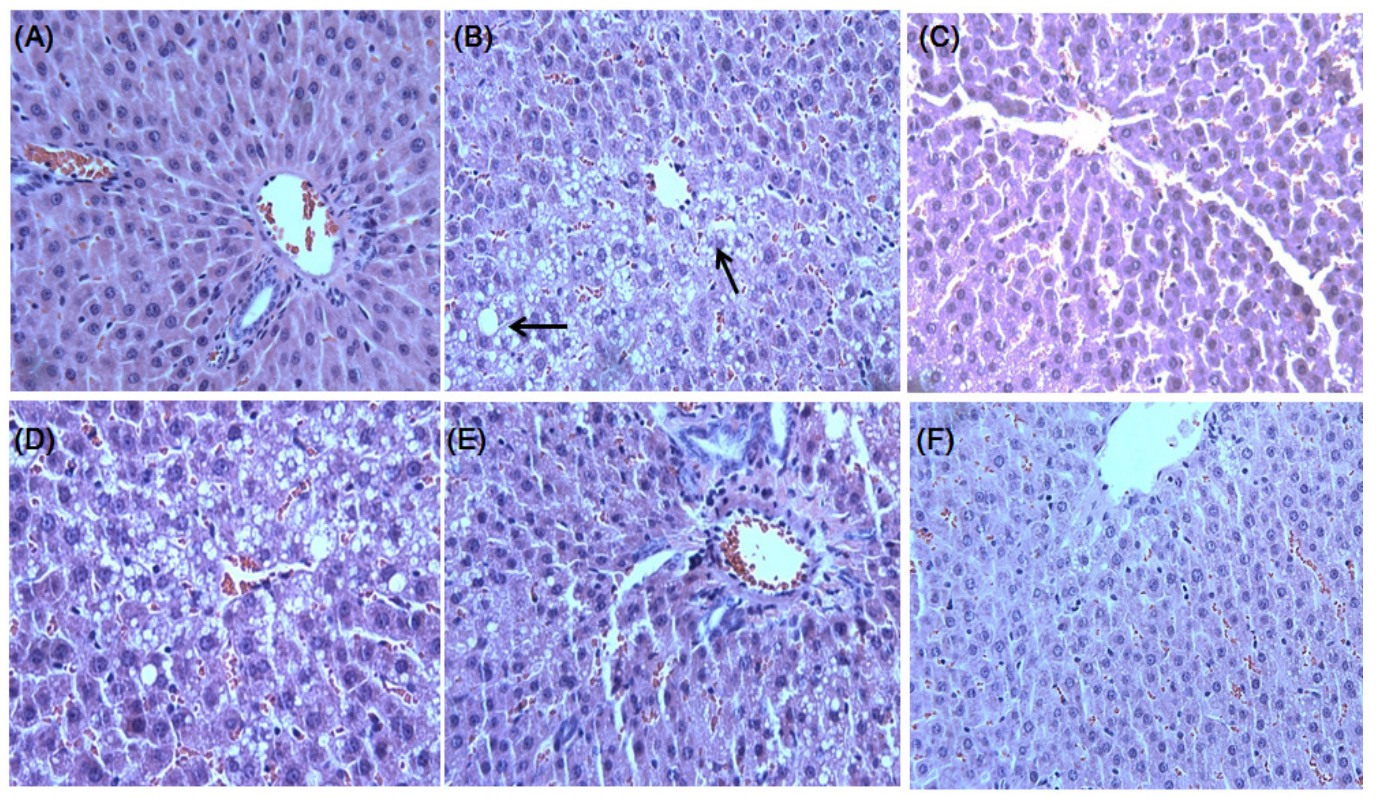

Figure 4. Effects of DCPE on final femur bone mineral density (BMD) (A), femur BMD changes (B), uterus index $(\mathbf{C})$ and organ index (D). Data are the mean $\pm \mathrm{SD}(n=12$ per group). $* p<0.05, * * p<0.01$ and $* * * p<0.001$, significant differences from the OVX-control group.

(A)

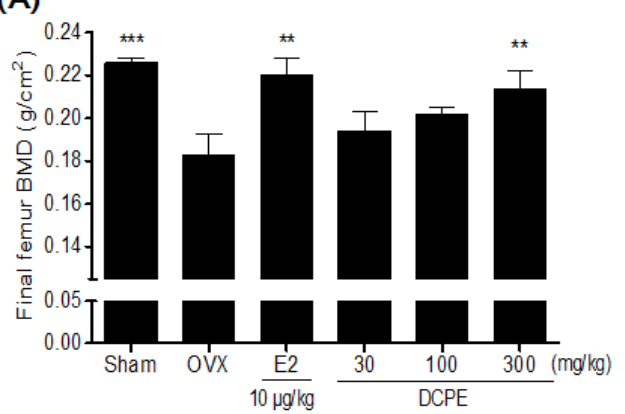

(C)

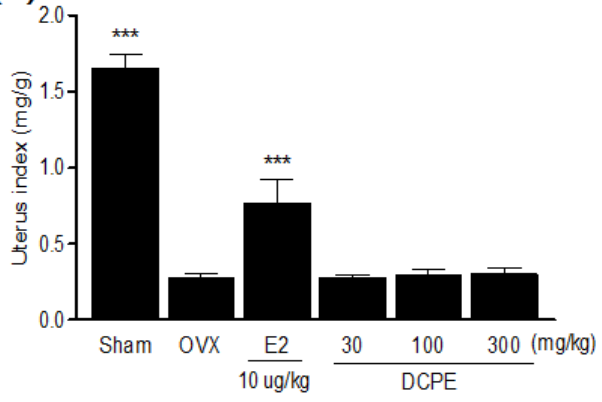

(B)

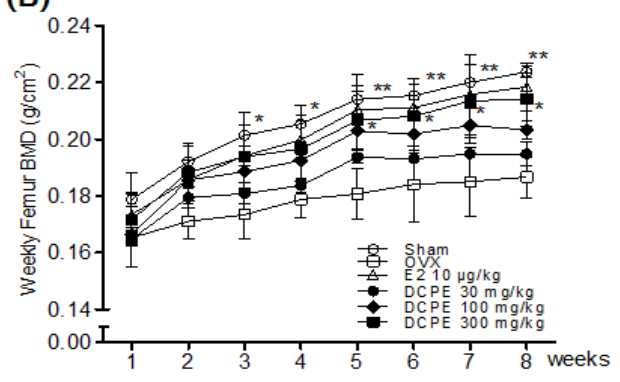

(D)

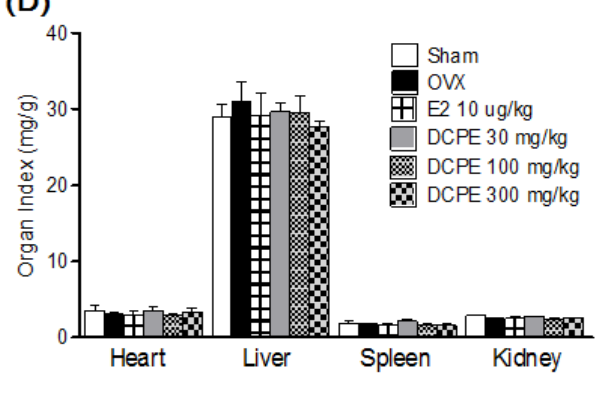




\subsection{Effect of DCPE on Bone Marker in OVX Rats}

In our previous results [20], alkaline phosphatase (ALP), telopeptides of collagen type I (CTx) and osteocalcin (OC) levels in the OVX-control group were significantly higher compared to the sham group. After eight weeks of treatments, the DCPE $300 \mathrm{mg} / \mathrm{kg}$ treated group displayed significantly lower ALP, CTx and OC levels compared to the OVX-control group (Figure 5). In case of serum estradiol levels, the DCPE treated groups were not significantly different from the OVX-control group (Figure 5A).

Figure 5. Effects of DCPE on serum estradiol (A), ALP (B), OC (C) and CTx (B) levels. Data are the mean $\pm \mathrm{SD}$ values ( $n=12$ per group). $* p<0.05 * * p<0.01$ and $* * * p<0.001$, significant differences from the OVX-control group.
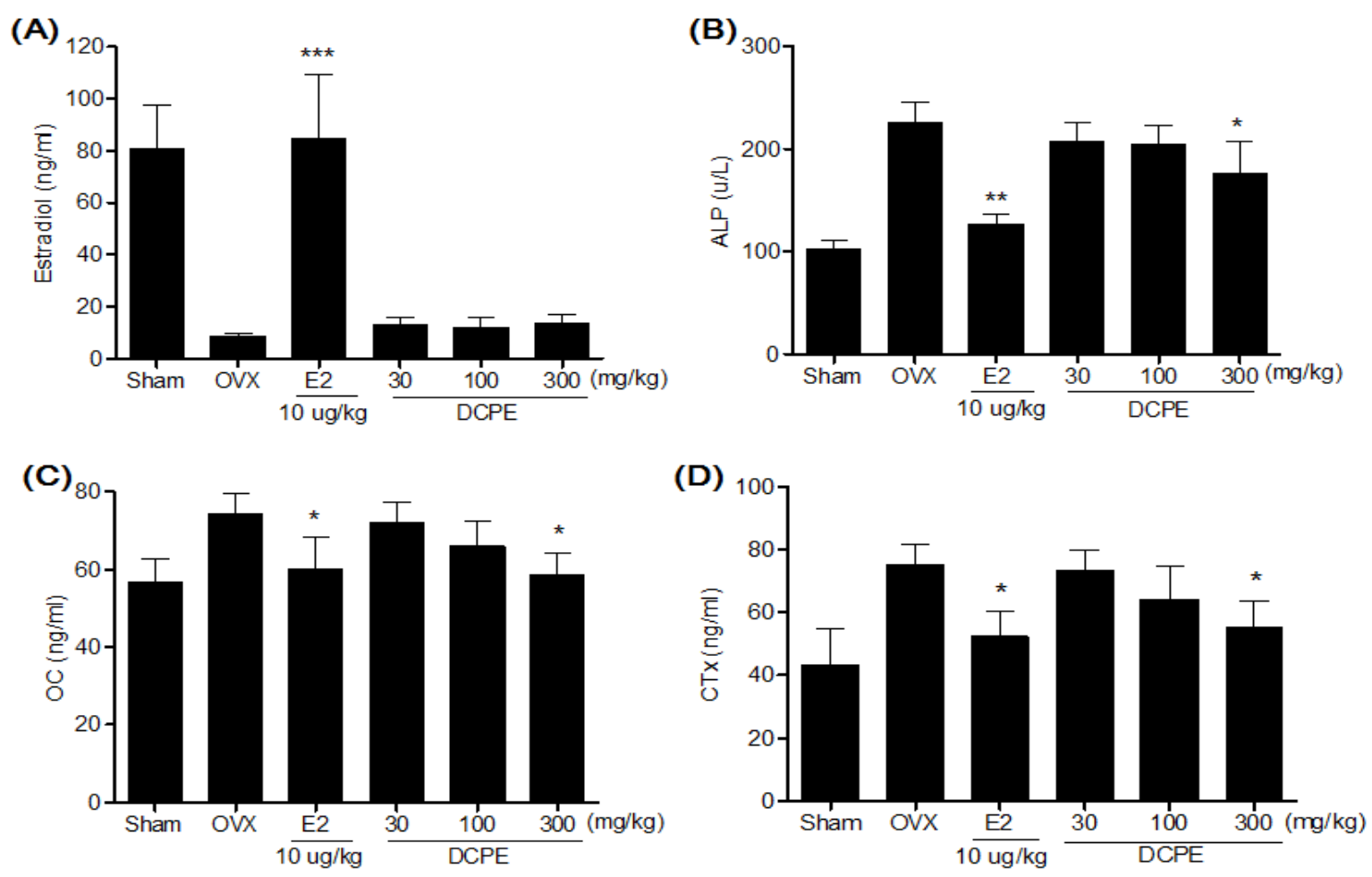

\subsection{Discussion}

Our results demonstrate that the treatment with high-dose DCPE for eight-weeks significantly decreased the BMD loss in the femur and inhibited the increase in lipoprotein levels compared to the OVX-control group. These findings indicate that DCPE induced multiple metabolic changes in a rat model of postmenopausal symptoms.

Bone loss caused by estrogen deficiency in both experimental animals and humans is primarily due to an increase in osteoclastic bone resorption [21]. OVX rats, which display most of the characteristics of human postmenopausal osteoporosis [19] have been widely used as a model for the evaluation of potential osteoporosis treatments [22].

Like previous reports, our OVX resulted in significant decrease in the femur BMD after eight weeks. This BMD loss was accompanied by a significant increase in bone remodeling, as evidenced by 
the enhanced bone turnover makers. Elevated alkaline phosphatase (ALP) in serum levels, the most widely used biochemical bone turnover marker [23], was observed in OVX rats. Moreover, an increase of telopeptides of collagen type I (CTx), correlates with bone-resorption and bone formation marker osteocalcin (OC) in serum levels were observed in OVX rats. These results were supported by Hertrampf et al. [24]. The treatment with DCPE $300 \mathrm{mg} / \mathrm{kg}$ for eight week significantly decreased the BMD loss in the femur. It was reflected by the decrease in ALP, CTx and OC serum levels compared to the OVX-control group; this effect may have been caused by the decreased bone resorption.

Park et al. [11] found that supplementation with C. unshiu peel extract resulted in a significant decrease in body weight gain, body fat mass and blood glucose level in type 2 diabetic db/db mice. This suggestion was supported by the anti-obesity effects of Citrus peel extracts in a high fat diet-induced obesity in vivo model accompanied by regulation of the mRNA expressions of genes involved in adipogenesis in the adipose tissue $[10,14]$. However, in our results, there was no significant difference in the body weight gain of the DCPE treated groups in OVX rats. OVX dramatically increases body weights, while E2 treatment presents completely normal levels [25]. Although the mechanisms by which OVX induces an increase in body weight are not clear, estrogen deficiency induced body fat accumulation and subsequently caused an increase in body weight [26]. Estrogen may be directly involved in rat energy metabolism by binding to ER within the abdominal and subcutaneous fat tissues [27]. Estrogen affects its activities by binding to different estrogen receptors (ERs), ER $\alpha$ and ER $\beta . E R \beta$ is more abundant than ER $\alpha$ in bone tissue while ER $\alpha$ is mainly distributed in reproductive cells. ER $\alpha$ is the dominant receptor mediating the most obvious effects of E2 in breast and uterus [28]. Oral administration of DCPE did not affect body weight gain, uterotrophic activity, and serum estradiol concentration in OVX rats. Consistent with our finding from the E2 treated group, the DCPE might have anti-osteoporotic effects in OVX rats, without the influence of hormones such as estrogen.

We found in current study that there was an obvious lipid metabolic disturbance in OVX rats. The TC and TG concentration in liver in OVX control group at eight weeks after surgery was found to be obviously higher than those in other groups. Although OVX increases TC and TG concentrations in liver, the increased insulin concentration in OVX rats may accelerate the dephosphorylation effect of the HMG-CoA reductase, which is a rate-limiting enzyme of the composition of cholesterol in liver [29]. Serum AST and ALT levels are clinically and toxicologically important indicators [30], and increase as a results of tissue damage caused by toxicants or disease conditions. It has also been reported that a high consumption of citrus flavonoids decreases the risk of coronary heart disease, given that naringin or hesperidin lowers serum cholesterol and triglycerides in rats [31]. Bok et al. [32] demonstrated that a mixture of naringin and hesperidin significantly lowered the levels of plasma and hepatic cholesterol and triglycerides as well as the HMG-CoA reductase activity in rats. In our results, DCPE caused no apparent liver toxicity, based on lower plasma AST and ALT. Moreover, DCPE exhibits a hepatoprotective effect, indicated with improved hepatic lipid contents in OVX rats. These results suggest that DCPE could regulate the lipid metabolic disturbance in liver in the OVX rats. 


\section{Experimental}

\subsection{Sample Preparation and HPLC Analysis}

Dried peel of $C$. unshiu was purchased from the Kapdang Co. (Seoul, Korea). The sample was identified by Dr. HeeSoon Shin and a voucher specimen (\#NP-1047) was deposited in the Functionality Evaluation Research Group, Korea Food Research Institute, Seoul, Korea. The dried peel of C. unshiu (300 g) was extracted separately with $70 \%$ ethanol $(3,000 \mathrm{~mL})$ for $3 \mathrm{~h}$ at $80{ }^{\circ} \mathrm{C}$ in a reflux apparatus. The extracts were filtered and concentrated under reduced pressure, and samples were lyophilized to yield a dark yellow powder. The yield of extracts was $20.1 \%$. The standardization of C. unshiu peel extracts was performed by a high performance liquid chromatography (HPLC) system equipped with a Waters 1525 pump, a 2707 auto sampler and a 2998 photodiode array detector (PDA) detector. The separation was achieved at $30^{\circ} \mathrm{C}$ on Waters Sunfire ${ }^{\mathrm{TM}} \mathrm{C} 18(250 \mathrm{~mm} \times 4 \mathrm{~mm}$ i.d., $5 \mu \mathrm{m}$ particle size) column. C. unshui peel extracts was monitored at $284 \mathrm{~nm}$ for hesperidin. The run time was set at $25 \mathrm{~min}$ and the flow rate was $1.0 \mathrm{~mL} / \mathrm{min}$ (sample injection volume was $10 \mu \mathrm{L}$ ). The mobile phases $\mathrm{A}$ and $\mathrm{B}$ were $1 \% \mathrm{H} 3 \mathrm{PO} 4(\mathrm{v} / \mathrm{v})$ and $\mathrm{CH} 3 \mathrm{CN}$, respectively. The gradient elution was as follows: 0-10 min 0\%-40\% solvent B, 10-15 min 40\%-100\% solvent B, 15-25 min $100 \%-0 \%$ solvent B. C. unshiu peel extracts was standardized to contain $21.7 \mathrm{mg} / \mathrm{g}$ hesperidin.

\subsection{Animals and Treatments}

Female Sprague-Dawley (SD) rats, 8-weeks-old, were purchased from Samtako (Gyeonggi-do, Korea). Animals were housed two rats per cage in an air-conditioned room at $23 \pm 1{ }^{\circ} \mathrm{C}, 55 \%-60 \%$ relative humidity and a $12 \mathrm{~h}$ light/dark cycle (07:00 lights on, 19:00 lights off) and were given a laboratory regular rodent diet. After acclimatization for 1 week, 9-week-old female SD rats were anesthetized with $2 \%$ of isoflurane and the ovaries were removed bilaterally. A sham operation, during which the ovaries were just touched with forceps, was performed on the sham group. One week after surgery, rats were divided into the six following treatment groups: sham + vehicle, OVX + vehicle, OVX $+17 \beta$-estradiol (E2, $10 \mu \mathrm{g} / \mathrm{kg}$ once daily, i.p.), OVX + DCPE (30, 100 and $300 \mathrm{mg} / \mathrm{kg}$, once daily, p.o.). E2 was dissolved in distilled water, with $1 \%$ dimethyl sulfoxide (DMSO) and $0.1 \%$ Tween 20. All groups were treated for eight weeks. During the experimental period, body weight and femur bone mineral density (BMD) were determined weekly. At the end of the treatment period, the rats fasted for $12 \mathrm{~h}$ and blood was collected via the abdominal aorta under $2 \%$ of isoflurane anaesthesia. Uterus tissue and other organs were dissected, washed with saline solution and weighed for analysis. Uterus and organ indexes $(\mathrm{mg} / \mathrm{g})$ were calculated by dividing the uterus and organ weights by the body weight. Liver tissues were collected for the experiments described later in this section. All animal experiments were carried out according to the guidelines of the Korea Food Research Institutional Animal Care and Use Committee.

\subsection{BMD Measurements}

The BMD of femur was measured by a PIXImus (GE Lunar PIXImus, GE Healthcare, Madison, WI, USA), dual energy X-ray absorptiometer (DXA), equipped with appropriate software for bone 
density assessment in small laboratory animals. Calibration of the instrument was conducted as recommended by the manufacturer. Quality control with BMD $\left(0.0553 \mathrm{~g} / \mathrm{cm}^{2}\right)$ and percentage fat composition (16.7\%) of the phantom were also performed each time the instrument was switched on. All rats were placed in the same direction.

\subsection{Serum Lipid, Estradiol and Bone Marker Analysis}

The serum samples were prepared by centrifugation of the collected blood samples $(1,013 \mathrm{~g}$ for $15 \mathrm{~min}$ at $4{ }^{\circ} \mathrm{C}$ ), then stored at $-80{ }^{\circ} \mathrm{C}$ for biochemical determinations. The serum concentrations of total cholesterol (TC), triglyceride (TG), high density lipoprotein-cholesterol (HDL-c), alanine transaminase (ALT), aspartate aminotransferase (AST) and alkaline phosphatase (ALP) were determined using an automatic analyzer (ADVIA 1650, Bayer, Tokyo, Japan). Serum LDL-c was estimated using Frieldwann's equation. Serum hormone level was determined by radioimmunoassay (RIA). The estradiol RIA was performed according to the instructions accompanying a Coat-a-Count kit (Diagnostic Products, Los Angeles, CA, USA). Serum concentrations of the bone formation marker, osteocalcin (OC), were assayed using a rat ELISA kit (Metra OC, Quidel Corporation, San Diego, CA, USA). Serum levels of telopeptides of collagen type I (CTx), which correlate with bone resorption, with high levels indicating excessive osteoclastic activity, were analyzed using commercial ELISA kits (Serum CrossLaps, Nordic Bioscience, Herlev, Denmark; Metra Serum Pyd, Quidel Corporation). The hepatic lipid was extracted using the procedure developed by Folch et al. [33]. TC and TG contents were determined using a commercial enzymatic kit (Asan Pharm. Co., Seoul, Korea).

\subsection{Liver Histological Analysis}

The liver tissues were divided into the tissue freezing medium (TBS, Durham, NC, USA) and then deep-frozen in liquid nitrogen for their preservation. The liver tissues were cut into $5 \mu \mathrm{m}$-thick sections using a Cryostat instrument (CM 1850; Leica, Heidelberg, Germany). The tissue sections were fixed in $10 \%$ neutral phosphate-buffered formalin solution. The lipids and nuclei of the liver cells were stained with hematoxylin and eosin $(\mathrm{H} \& \mathrm{E})$. A diagnosis of fatty liver was made based on the presence of macro- or micro-vesicular fat in $>5 \%$ of the hepatocytes in a given slide.

\subsection{Statistical Analysis}

All data were presented as the mean \pm standard deviation (SD). The effects of different treatments were compared by one-way ANOVA test, followed by the post-hoc Tukey_test for multiple comparisons using GraphPad Prism 5 (GraphPad Software Inc., La Jolla, CA, USA). $p<0.05$ was considered statistically significant.

\section{Conclusions}

In conclusion, our results demonstrated that DCPE has multiple metabolic benefits in OVX rats. The treatment of DCPE led to decreased serum lipoprotein levels and significantly improved femur BMD, without elevating the serum levels of the liver enzymes, AST and ALT. Taken together, our 
findings suggest that DCPE has the potential to improve both lipid and bone metabolism without influencing hormones such as estrogen in OVX rats.

\section{Acknowledgments}

This study was supported by a research grant from the Korea Food Research Institute.

\section{Author Contributions}

D.W. Lim and Y. Lee performed experiments and analyzed the data. D.W. Lim and Y.T. Kim conceived and designed the study and wrote the paper.

\section{Conflicts of Interest}

The authors declare no conflict of interest.

\section{References}

1. Pinkerton, J.V.; Guico-Pabia, C.J.; Taylor, H.S. Menstrual cycle-related exacerbation of disease. Am. J. Obstet. Gynecol. 2010, 202, 221-231.

2. Greendale, G.A.; Sowers, M. The menopause transition. Endocrinol. Metab. Clin. North Am. 1997, 26, 261-277.

3. Nelson, H.D.; Humphrey, L.L.; Nygren, P.; Teutsch, S.M.; Allan, J.D. Postmenopausal hormone replacement therapy: Scientific review. JAMA 2002, 288, 872-881.

4. Pradhan, A.D.; Manson, J.E.; Rossouw, J.E.; Siscovick, D.S.; Mouton, C.P.; Rifai, N.; Wallace, R.B.; Jackson, R.D.; Pettinger, M.B.; Ridker, P.M. Inflammatory biomarkers, Hormone replacement therapy, and incident coronary heart disease: Prospective analysis from the women's health initiative observational study. JAMA 2002, 288, 980-987.

5. Hedelin, M.; Klint, A.; Chang, E.T.; Bellocco, R.; Johansson, J.E.; Andersson, S.O.; Heinonen, S.M.; Adlercreutz, H.; Adami, H.O.; Gronberg, H.; et al. Dietary phytoestrogen, serum enterolactone and risk of prostate cancer: The cancer prostate sweden study (sweden). Cancer Causes Control 2006, 17, 169-180.

6. Taku, K.; Umegaki, K.; Sato, Y.; Taki, Y.; Endoh, K.; Watanabe, S. Soy isoflavones lower serum total and ldl cholesterol in humans: A meta-analysis of 11 randomized controlled trials. Am. J. Clin. Nutr. 2007, 85, 1148-1156.

7. Ma, D.F.; Qin, L.Q.; Wang, P.Y.; Katoh, R. Soy isoflavone intake increases bone mineral density in the spine of menopausal women: Meta-analysis of randomized controlled trials. Clin. Nutr. 2008, 27, 57-64.

8. Shedd-Wise, K.M.; Alekel, D.L.; Hofmann, H.; Hanson, K.B.; Schiferl, D.J.; Hanson, L.N.; van Loan, M.D. The soy isoflavones for reducing bone loss study: 3-yr effects on pqct bone mineral density and strength measures in postmenopausal women. J. Clin. Densitom. 2011, 14, $47-57$. 
9. Chilibeck, P.D.; Vatanparast, H.; Pierson, R.; Case, A.; Olatunbosun, O.; Whiting, S.J.; Beck, T.J.; Pahwa, P.; Biem, H.J. Effect of exercise training combined with isoflavone supplementation on bone and lipids in postmenopausal women: A randomized clinical trial. J. Bone Miner. Res. 2013, $28,780-793$.

10. Kang, S.I.; Shin, H.S.; Kim, H.M.; Hong, Y.S.; Yoon, S.A.; Kang, S.W.; Kim, J.H.; Kim, M.H.; Ko, H.C.; Kim, S.J. Immature citrus sunki peel extract exhibits antiobesity effects by beta-oxidation and lipolysis in high-fat diet-induced obese mice. Biol. Pharm. Bull. 2012, 35, 223-230.

11. Park, H.J.; Jung, U.J.; Cho, S.J.; Jung, H.K.; Shim, S.; Choi, M.S. Citrus unshiu peel extract ameliorates hyperglycemia and hepatic steatosis by altering inflammation and hepatic glucose- and lipid-regulating enzymes in db/db mice. J. Nutr. Biochem. 2013, 24, 419-427.

12. Oh, Y.C.; Cho, W.K.; Jeong, Y.H.; Im, G.Y.; Yang, M.C.; Hwang, Y.H.; Ma, J.Y. Anti-inflammatory effect of citrus unshiu peel in lps-stimulated raw 264.7 macrophage cells. Am. J. Chin. Med. 2012, 40, 611-629.

13. Kim, D.K.; Lee, K.T.; Eun, J.S.; Zee, O.P.; Lim, J.P.; Eum, S.S.; Kim, S.H.; Shin, T.Y. Anti-allergic components from the peels of citrus unshiu. Arch. Pharm. Res. 1999, 22, 642-645.

14. Yang, G.; Lee, J.; Jung, E.D.; Ham, I.; Choi, H.Y. Lipid lowering activity of citri unshii pericarpium in hyperlipemic rats. Immunopharmacol. Immunotoxicol. 2008, 30, 783-791.

15. Lee, S.; Ra, J.; Song, J.Y.; Gwak, C.; Kwon, H.J.; Yim, S.V.; Hong, S.P.; Kim, J.; Lee, K.H.; Cho, J.J.; et al. Extracts from citrus unshiu promote immune-mediated inhibition of tumor growth in a murine renal cell carcinoma model. J. Ethnopharmacol. 2011, 133, 973-979.

16. Lu, Y.; Zhang, C.; Bucheli, P.; Wei, D. Citrus flavonoids in fruit and traditional chinese medicinal food ingredients in china. Plant Food. Hum. Nutr. 2006, 61, 57-65.

17. Bicu, I.; Mustata, F. Cellulose extraction from orange peel using sulfite digestion reagents. Bioresource Technol. 2011, 102, 10013-10019.

18. Deyhim, F.; Garica, K.; Lopez, E.; Gonzalez, J.; Ino, S.; Garcia, M.; Patil, B.S. Citrus juice modulates bone strength in male senescent rat model of osteoporosis. Nutrition 2006, 22, 559-563.

19. Jee, W.S.; Yao, W. Overview: Animal models of osteopenia and osteoporosis. J. Musculoskelet. Neuronal Interact. 2001, 1, 193-207.

20. Lim, D.W.; Kim, J.G.; Lee, Y.; Cha, S.H.; Kim, Y.T. Preventive effects of eleutherococcus senticosus bark extract in ovx-induced osteoporosis in rats. Molecules 2013, 18, 7998-8008.

21. Hoegh-Andersen, P.; Tanko, L.B.; Andersen, T.L.; Lundberg, C.V.; Mo, J.A.; Heegaard, A.M.; Delaisse, J.M.; Christgau, S. Ovariectomized rats as a model of postmenopausal osteoarthritis: Validation and application. Arthritis Res. Ther. 2004, 6, R169-R180.

22. Lelovas, P.P.; Xanthos, T.T.; Thoma, S.E.; Lyritis, G.P.; Dontas, I.A. The laboratory rat as an animal model for osteoporosis research. Comp. Med. 2008, 58, 424-430.

23. Nishizawa, Y.; Nakamura, T.; Ohta, H.; Kushida, K.; Gorai, I.; Shiraki, M.; Fukunaga, M.; Hosoi, T.; Miki, T.; Chaki, O.; et al. Guidelines for the use of biochemical markers of bone turnover in osteoporosis (2004). J. Bone Miner. Metab. 2005, 23, 97-104.

24. Hertrampf, T.; Schleipen, B.; Offermanns, C.; Velders, M.; Laudenbach, U.; Diel, P. Comparison of the bone protective effects of an isoflavone-rich diet with dietary and subcutaneous administrations of genistein in ovariectomized rats. Toxicol. Lett. 2009, 184, 198-203. 
25. Devareddy, L.; Khalil, D.A.; Smith, B.J.; Lucas, E.A.; Soung, D.Y.; Marlow, D.D.; Arjmandi, B.H. Soy moderately improves microstructural properties without affecting bone mass in an ovariectomized rat model of osteoporosis. Bone 2006, 38, 686-693.

26. Dang, Z.C.; van Bezooijen, R.L.; Karperien, M.; Papapoulos, S.E.; Lowik, C.W.G.M. Exposure of ks483 cells to estrogen enhances osteogenesis and inhibits adipogenesis. J. Bone Miner. Res. 2002, 17, 394-405.

27. Joyner, J.M.; Hutley, L.J.; Cameron, D.P. Estrogen receptors in human preadipocytes. Endocrine 2001, 15, 225-230.

28. Hewitt, S.C.; Korach, K.S. Oestrogen receptor knockout mice: Roles for oestrogen receptors alpha and beta in reproductive tissues. Reproduction 2003, 125, 143-149.

29. Wang, J.F.; Guo, Y.X.; Niu, J.Z.; Liu, J.; Wang, L.Q.; Li, P.H. Effects of radix puerariae flavones on liver lipid metabolism in ovariectomized rats. World J. Gastroenterol. 2004, 10, 1967-1970.

30. Radi, Z.A.; Koza-Taylor, P.H.; Bell, R.R.; Obert, L.A.; Runnels, H.A.; Beebe, J.S.; Lawton, M.P.; Sadis, S. Increased serum enzyme levels associated with kupffer cell reduction with no signs of hepatic or skeletal muscle injury. Am. J. Pathol. 2011, 179, 240-247.

31. Shin, Y.W.; Bok, S.H.; Jeong, T.S.; Bae, K.H.; Jeoung, N.H.; Choi, M.S.; Lee, S.H.; Park, Y.B. Hypocholesterolemic effect of naringin associated with hepatic cholesterol regulating enzyme changes in rats. Int. J. Vitam. Nutr. Res. 1999, 69, 341-347.

32. Bok, S.H.; Lee, S.H.; Park, Y.B.; Bae, K.H.; Son, K.H.; Jeong, T.S.; Choi, M.S. Plasma and hepatic cholesterol and hepatic activities of 3-hydroxy-3-methyl-glutaryl-coa reductase and acyl coa: Cholesterol transferase are lower in rats fed citrus peel extract or a mixture of citrus bioflavonoids. J. Nutr. 1999, 129, 1182-1185.

33. Folch, J.; Lees, M.; Sloane Stanley, G.H. A simple method for the isolation and purification of total lipides from animal tissues. J. Biol. Chem. 1957, 226, 497-509.

Sample Availability: Samples of the C. unshiu peel extracts are available from the authors.

(C) 2014 by the authors; licensee MDPI, Basel, Switzerland. This article is an open access article distributed under the terms and conditions of the Creative Commons Attribution license (http://creativecommons.org/licenses/by/3.0/). 